\title{
COMPARISON OF OPTICAL METHODS FOR CHARACTERIZATION OF GLASS MOSAIC WEATHERING
}

\author{
Vladimir Kocour*, Veronika Petranova, Jaroslav Valach \\ Institute of Theoretical and Applied Mechanics, Prosecka 76, 19000 Prague, Czech Republic \\ * corresponding author: kocour@itam.cas.cz
}

\begin{abstract}
The work presented in this paper is a part of research project supported by Ministry of Culture and devoted to conservation of precious mosaic of "Last Judgement" on St. Vitus Cathedral in Prague. The aim of the project is to enhance external protective polymeric coating on glass tesserae of mosaic and also to develop optical method for assessment of coating's surface conditions. The paper concentrates on comparison of various methods for surface evaluation assesses their advantages and disadvantages and also discusses their suitability for long term monitoring of coating state, namely reflectometry, scanning probe microscopy, nanoindentation, white-light interferometry, and scanning electron microscopy. It is found that white-light interferometry and reflectometry methods are the most suitable ones for monitoring purposes.
\end{abstract}

KEYwORDS: surface, reflectance, photometry, roughness, glass mosaic.

\section{INTRODUCTION}

This study is a part of extensive research project devoted to protection of medieval glass mosaic found on Southern entrance to St. Vitus cathedral on Prague Castle. Finished at the end of 14th century, the Last Judgement Mosaic at south wall of St. Vitus Cathedral in Prague Castle complex belongs to the oldest outdoor glass mosaics in the Czech Republic (see [1] and 2] for more details on mosaic history and conservation).

Although glass is being considered as a stable material, the reality is more complicated, especially if we are interested in long-time properties of the glass. Historical glass is different from contemporary in chemical composition and production technology. Therefore, its resistance to elements is generally lower and gradual degradation takes part. As tesserae of glass mosaics are exposed to various harmful effects like temperature fluctuation, rain, frost, chemicals, their durability can be improved with some protection coating [3]. Based on previous restoration works, the glass is protected by a two-layer polymeric coating system and an additional new layer is now under development. But also the resistance of the coating itself is limited and has to be carefully selected. Especially for monitoring of the state of this new layer, a novel monitoring method is being developed.

\section{Specimen Treatment And CHARACTERIZATION}

\subsection{Artificial Weathering of Specimens}

As a first step, glass specimens - microscopy slides dip coated with the polymeric coating were prepared and artificially weathered to observe and measure effects of degradation process. One half of every slide was covered with the investigated protective layer, while second half was left uncoated and unprotected. Changes in appearance were measured after one week, two weeks, three weeks, four weeks, five weeks, and six weeks of artificial weathering of high humidity and temperature [4].

\subsection{Sufrace Characterization of SPECIMENS}

It is known that surface roughness and angular reflectivity are closely related to surface topography and can be used to characterize the degree of degradation. Changes of the surface were visible and the deployed optical and mechanical methods showed, that these changes are also measurable [5] 6]. One variable which is used for characterization of surface topography is arithmetic roughness. Arithmetical roughness is defined as

$$
R_{a}=\frac{\sum_{i=1}^{N}\left|Z_{i}-\bar{Z}\right|}{N},
$$

where $N$ is the number of points in the area, where roughness is calculated, $Z_{i}$ is the measured $Z$-coordinate for each individual point, $\bar{Z}$ is the arithmetic mean of $Z_{i}$ of all points in the area. Our measurement shows that this variable increases with time of artificial weathering. Another useful measure explained later is the change of reflectivity of surface from "specular" to "Lambertian", i. e. evenly matt. The reflectance property enables to determine quantitatively "position" of surface on gloss to matt scale for given wavelength (surface may appear rough at shortwaves while being glossy at longwaves of electro-magnetic radiation). This position can be used to indicate surface roughness. Various methods 
were used to characterize changes occurring on the surface and inside the weathered coating. The changes result in visual degradation, loss of transparency and gloss. To study these surface alterations, we used digital microscopy, scanning probe microscopy (SPM), scanning electron microscopy (SEM), interferometry in white light and angle depended reflectometry. The following chapter concentrates on selection of suitable non-invasive methods for assessment and monitoring of coating properties.

\section{Used Optical and Mechanical METHODS}

\subsection{Digital Microscope}

A 3D microscope (Hirox KH-7700) with focus stacking technique was used. This technique overcomes the limitation of optical microscopy of shallow depth of focus by combining multiple images with different focus. The visual changes documented by this digital microscope with enhanced depth of focus can be associated with roughening of the coating surface due to chemical and physical effects. One can observe snowflake like surface features, formation of rounded protrusions, ridges and valleys as surface "wrinkles". The changes (see Fig. 1) are visually assessed by naked eye as gradual decrease of gloss and also as diffuse appearance of the surface.

\subsection{NANOINDENTER WITH SPM}

Degradation of coating surface was also studied by nanoindenter (Hysitron TI-750). It was found that hardness increases significantly with duration of artificial weathering. SPM technique is integrated into the device, therefore the microtopography of the coating can be analyzed as well. Increase of surface irregularities and overall roughness was observed as demonstrated in Fig. 2. SPM is not limited in its resolution to features comparable to wavelength of visible light as optional microscopy and can be used to measure fine surface microtopography. On the other hand, a significant drawback of this technique is the horizontal size of analyzed area, which is limited to $80 \mu \mathrm{m}$. Therefore the investigated area cannot be considered representative.

\subsection{Scanning Electron Microscopy}

The scanning electron microscope Mira II. (Tescan, Brno, Czech Republic) was used for observation. However SEM overcomes the limitation of optical microscope in terms of resolution and depth of filed. In order to observe the coating in SEM, it is necessary to add a thin conductive layer (several nanometers of carbon or gold). Therefore the method cannot be considered as non-invasive technique. A backscattered electron detector and a secondary electron detector are predominantly used to record a signal and form an image of observed surface. Contrast in former is based on difference of atomic number in viewfield, while the topography is mostly recorded by the later. As seen on Fig. 3 the only atomic contrast is presence of dust particles (upper part) and as concerns the topography of initial coating's surface, this technique renders it flat (lower part of figure).

\subsection{White-Light InTERFEROMETRY}

White-light interferometry is an interferometric methods. Interferometric methods use interference of light. Interferometric devices are often constructed as Michelson interferometer. The first experiments with white-light interferometry have been done by A. A. Michelson [7]. However, the method became practically usable about 20 years ago in connection with the development of computational data processing. The name of this method is a bit improper. A better name would be "interferometry in polychromatic light" because the used light does not need to seem as "white" for human eye, and it is not necessary so that the light must be visible. A light source of time-incoherent radiation with continuous spectrum is used for this method. Light bulb, LED diode or super-luminescence diode can be such a light source. It is being utilized that coherence length of the radiation is short, and interference effect occurs only if difference of optical paths in the interferometer is comparable with the coherence length [8].

The measured specimen is situated in one arm. Reference mirror is situated in second arm. The specimen is equipped with a fine linear stage. Camera recording interfered light detects a series of snaps while the specimen moves on the stage. Graphical dependence of intensity of detected radiation (light) is called correlogramme. The serie of snaps is then processed with help of various methods, usually Hilbert transformation [9]. The result of this processing is the maximum of envelope of the correlogramme. Position of maximum of the correlogramme is equal to $z$-coordinate (height coordinate) of measured point.

We measured artificially weathered microscope slides coated with protective layer with Zygo white-light interferometer (Fig. 4). Correspondence between roughness and weathering is then summarized in Fig. 5. It shows that as weathering continues, the surface roughness increases.

\subsection{RefleCtometry}

Surfaces of real objects can be divided to three groups: 1. optically smooth, 2 . transition, 3. optically raw. For optically smooth surfaces $h<\lambda / 4$ and $\Delta \varphi<\pi$ in a cell of resolution. For optically raw surfaces $h>\lambda / 2$ and $\Delta \varphi>2 \pi$, where $h$ is relief height, $\lambda$ is wavelength of the radiation and $\Delta \varphi$ is phase shift detected in a cell of resolution. The transition surfaces have properties as smooth or raw surfaces in dependence of conditions and behave variously. A 

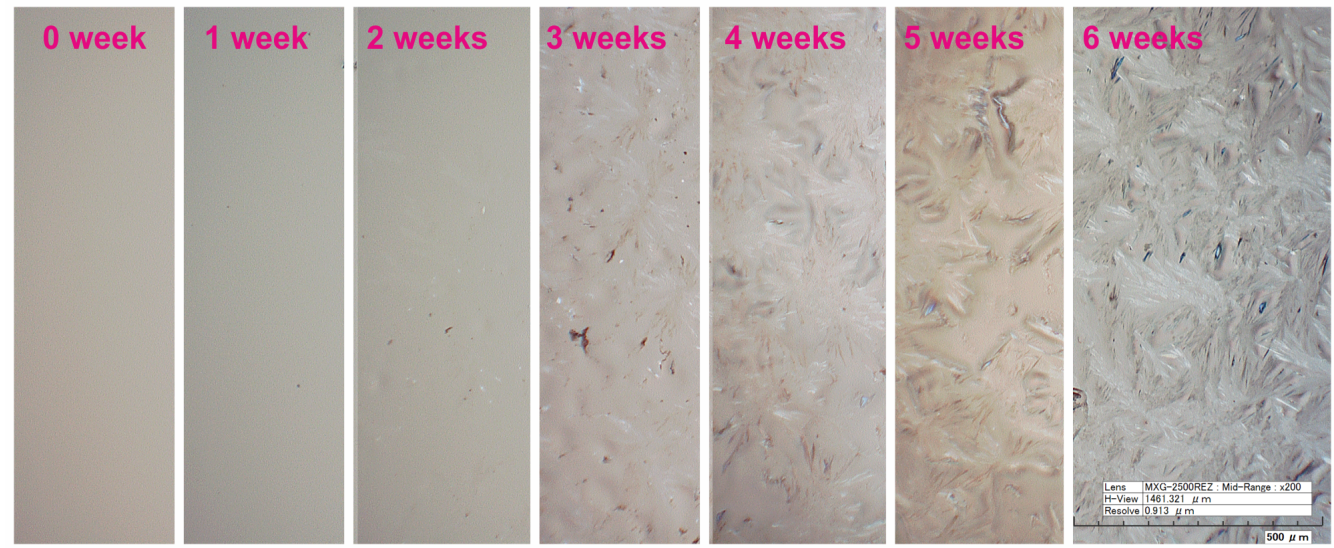

FiguRE 1. Digital microscope depiction of progressing surface degradation.

$\mathrm{OW}, \Delta \mathrm{z}=0.35 \mu \mathrm{m}$

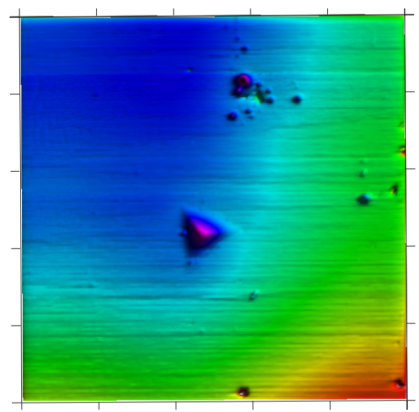

$3 \mathrm{~W}, \Delta \mathrm{z}=1.15 \mu \mathrm{m}$

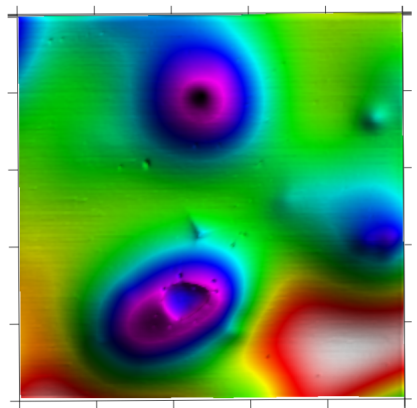

$80 \mu \mathrm{m}$

FiguRE 2. Gradual change of coating's surface measured by Scanning probe microscopy mode in nanoindenter. Color coded vertical scale changes from image to image, thus height difference between the lowest and highest point is given.

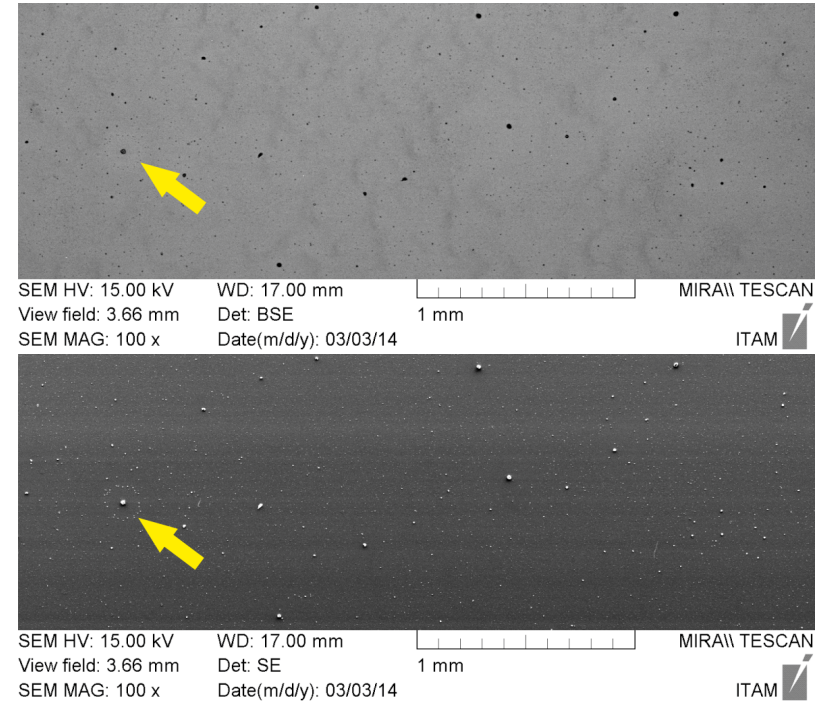

FIGURE 3. SEM image of the unweathered coating using (a) the BSE detector and (b) the SE detector.

quite general access includes a possibility of reflection of incidence ray out of incidence plane. Reflected radiation is completely described with bidirectional reflectance distribution function (BRDF) [10] [11.
Most of surfaces (except special cases like crocidolite, chatoyant glitter etc.) behaves "more orderly"; it is sufficient to study its reflectivity properties in plane of incidence ray.

For angle dependent reflectometry, we have designed and built a programmable automatic device. Reflectometry, in our interpretation, is measuring of relative intensity of reflected light in dependence on incidence angle $\phi$ and angle of observation $\varepsilon$, therefore the device is capable to create a two-dimensional map of relative intensity of reflected radiation in dependence on incidence angle and angle of observation ". As follows from 4 , where the relative intensity is expressed as false colour [12, the more weathered surface the more dispersed peak in reflectivity map can be found. Although this expression is more qualitative than quantitative, it is visible from such a map, how studied surface is specular or matt. The map shows relative intensity of light measured by reflectometer for given pair of incidence and observation angles $(\phi, \varepsilon)$, Fig. 6 . The maxima are naturally found for conditions, where the two angles are equal. Increase in coating's surface roughness is associated with widening of maximum's profile. 


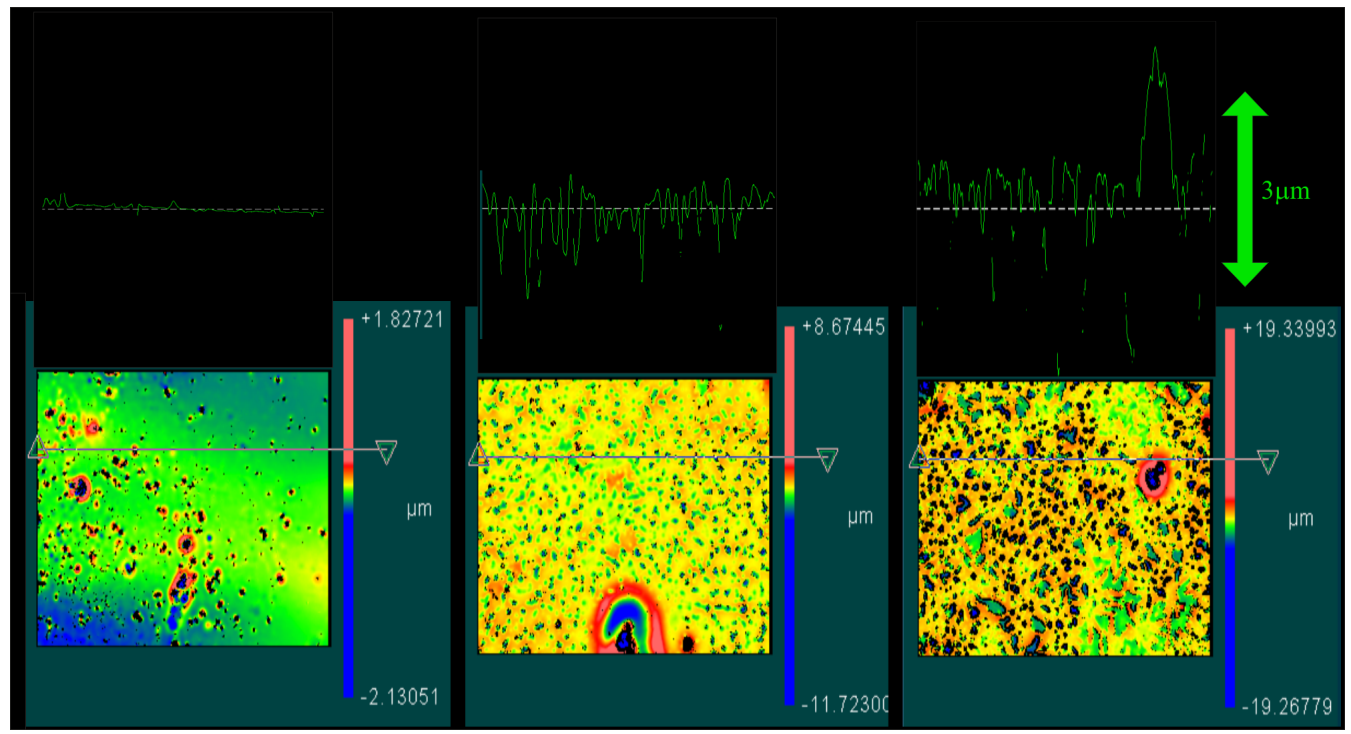

FigURE 4. Three artificially weathered specimens of glass covered with protective layer: 0 weeks (non-weathered), 3 weeks, and 5 weeks old. Above: horizontal profiles, below: false color height maps. Field of view $3.53 \times 2.56 \mathrm{~mm}$.

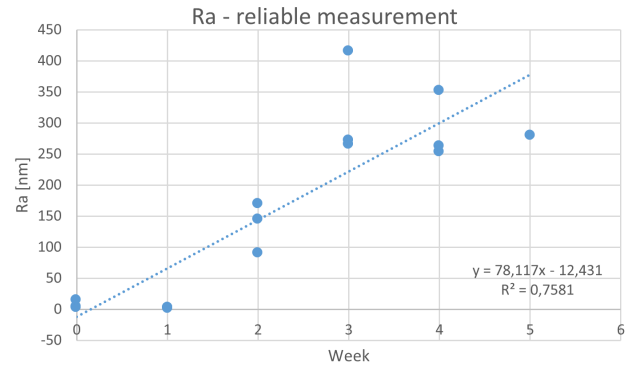

FiguRE 5. Relation between area roughness and duration of accelerated weathering of coating.

\section{Discussion And Conclusions}

Several methods capable of analyzing surface properties were applied in this presented comparative study. The major goal was to evaluate advantages and disadvantages of these methods and select the most suitable one for documentation and quantitative characterization of surface degradation. These methods will be exploited for long-term monitoring of protective coating of glass mosaic.

It was confirmed that optical methods can be used to quantify changes the changes induced by the degradation of polymeric coating induced by accelerated weathering.

It was found that the necessity to cover the surface of the specimen with a conductive layer excludes SEM from further consideration, because this additional layer alters the specimen properties. The applied technique has to be non-invasive, if long-term monitoring has to be achieved. SPM correctly indicates the surface topography changes, but the area studied was too small to be representative for the whole coating. Digital microscope can document coating modifications as weathering progresses. But in long-term monitoring based on observation of microscopy slides, there is a possibility the results would be hindered by degradation of glass below coating as the digital microscope is the only method in compared set that relies on transparency of the set microscopy slide - coating. Therefore methods quantifying the coating roughness as a measure of its degradation are the best candidates for reliable monitoring. A suitable method is direct roughness measurement as in white light interferometry or indirect expression of roughness in surface reflectivity as measured by reflectometer.

\section{ACKNOWLEDGEMENTS}

This research is supported by the Czech Ministry of Culture project \#DF12P01OVV017 "Technology of maintenance and conservation of the Last Judgment mosaic and methods of medieval and archaeological glass conservation".

Authors also thank to Dr. Vítů from Faculty of transportation sciences of the Czech Technical University for performing measurement on white light interferometer and to Dr. Šašek from CET Telč for nanoindentation.

\section{REFERENCES}

[1] J. Valach, V. Kocour, M. Černý. Investigation of Optical Properties of Protective Layer for Glass Medieval Mosaic of Last Judgement from St. Vitus Cathedral in Prague. In The 23rd International Congress on Glass, Book of Abstracts. Praha, Česká sklářská společnost, 2013. pp. 154-155. ISBN 978-80-904044-3-4.

[2] F. Piqué, D. Stulík. Conservation of the Last Judgment Mosaic, St. Vitus Cathedral, Prague. Los Angeles, Getty Conservation Institute. (2004).

[3] B. Holubová, Z. Zlámalová Cílová, I. Kučerová and M. Zlámal: Weatherability of hybrid organic-inorganic silica protective coatings on glass, Progress in Organic Coatings, 88, 172-180, (2015) 

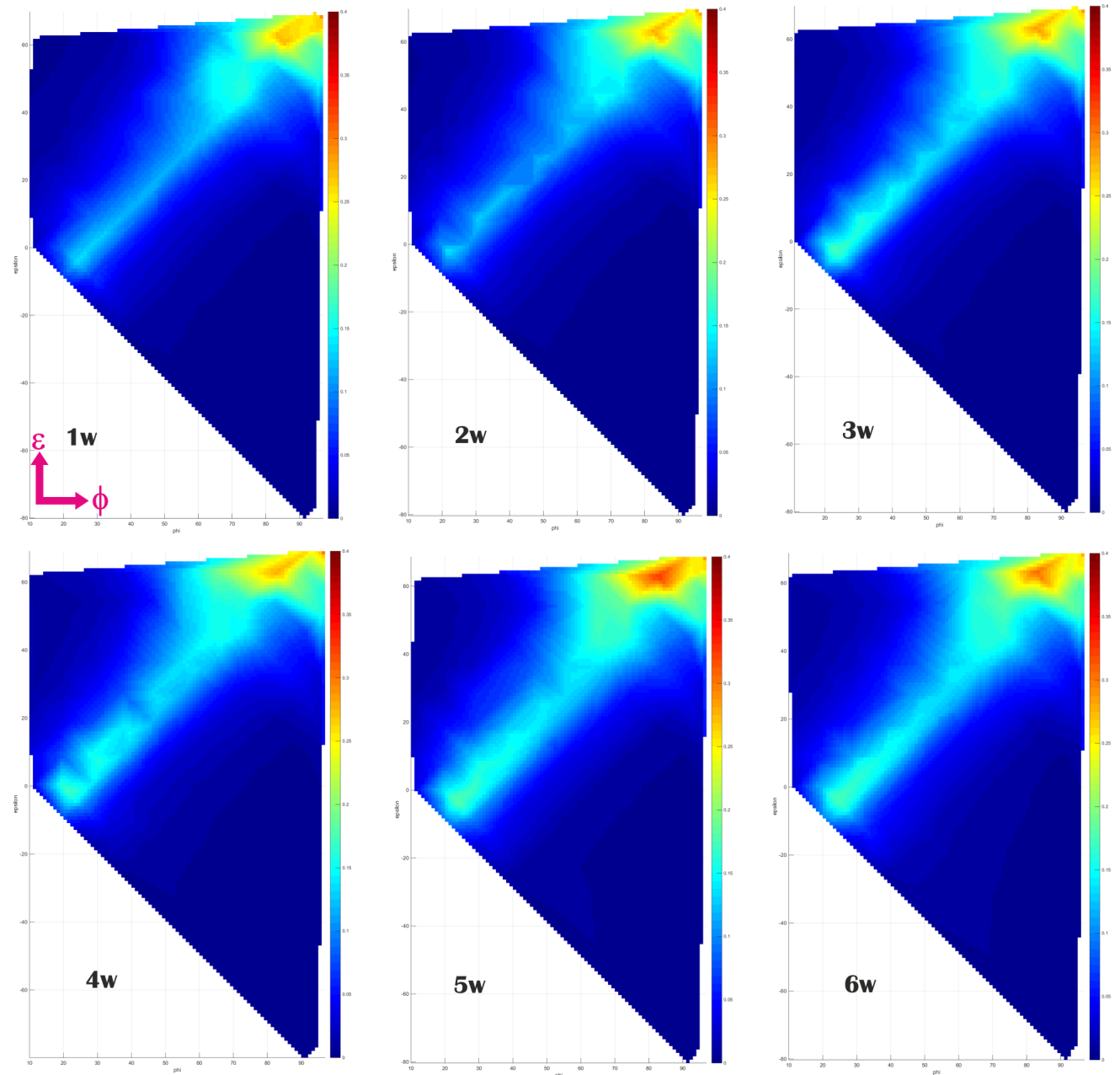

FIGURE 6. Series of surface reflectance representations demonstrating effect of accelerated weathering on optical properties of coating.

[4] L.F.E. Jacques, Accelerated and outdoor/natural exposure testing of coatings, Prog. Polym. Sci., 25 (9) (2000), pp. 1337-1362, DOI:10.1016/S0079-6700(00)00030-7

[5] H.E. Bennett, J.O. Porteus, Relation between surface roughness and specular reflectance at normal incidence, J. Opt. Soc. Am., 51 (1961), p. 123

[6] McKnight, M.E., Martin, J.W., Advanced methods and models for describing coating appearance, (1998) Progress in Organic Coatings, 34 (1-4), pp. 152-159.

[7] A. A. Michelson. Détermination Experimentale de la Valeur du Metre des Longeurs d'Ondes Lumineuses, Trav. Mem. Bur. Int. Poids Mes. 11, 1-42 (1895).

[8] Peter de Groot, Leslie Deck, Surface profiling by analysis of white-light interferograms in the spatial frequency domain, J. of Modern Optics, 42, Issue 2, pp 389-401, 1995

[9] R. Onodera, H. Watanabe, Y. Ishii. Interferometric Phase-Measurement Using a One-Dimensional Discrete Hilbert Transform, Optical Review, 12, (2005). DOI:10.1007/s10043-005-0029-7
[10] V. Kocour, J. Bryscejn, D. Vrba, J. Valach. Determination of Optical Properties of Surfaces for Use in Cultural Heritage Protection. In O. Jiroušek, D. Kytýřr. (ed.). Experimental Methods and Numerical Simulation in Engineering Sciences. Praha: Czech Technical University, Faculty of Transportation Sciences, 2012, pp. 15-18. ISBN 978-80-01-05062-0.

[11] J. Valach, V. Petráňová, D. Majtás, V. Kocour. Surface Degradation of Coating Measured by Means of Emissivity Change. In e-proceedings of Quantitative infrared thermography, Bordeaux, University of Bordeaux, 2014.

http://www.qirt.org/archives/qirt2014/QIRT

[12] V. Kocour, J. Valach. Semiautomatic Surface Reflectance Measurement for Monitoring of Material Weathering. In D. Kytýř, P. Zlámal, M. Růžička. (ed.). Proceedings of XIIIth youth symposium on experimental solid mechanics. Praha: Czech Technical University in Prague, Faculty of Transportation Sciences, 2014, pp. 56-60. ISBN 978-80-01-05556-4. 